
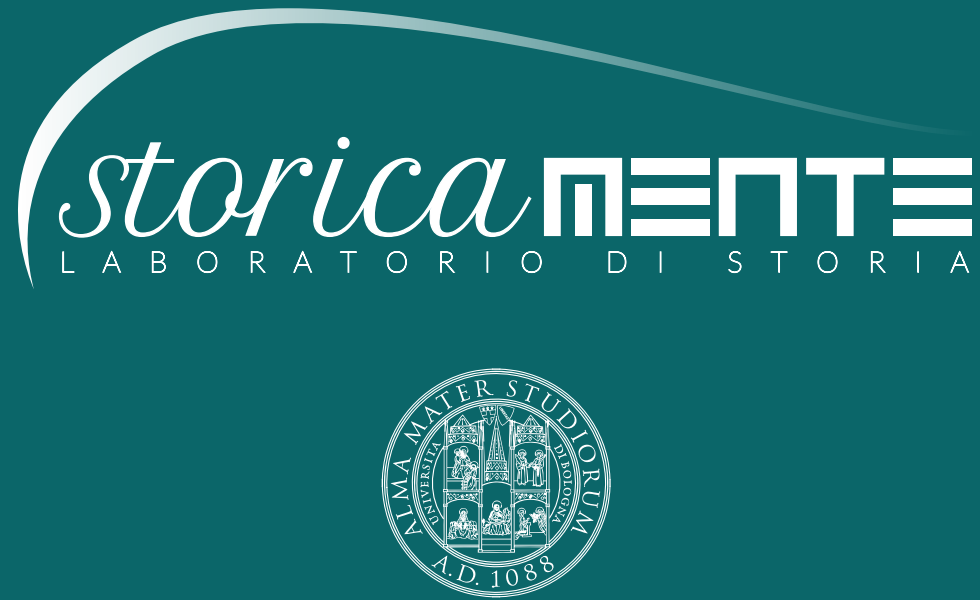

ALMA MATER STUDIORUM

Università di Bologna

Dipartimento di Storia Culture Civiltà

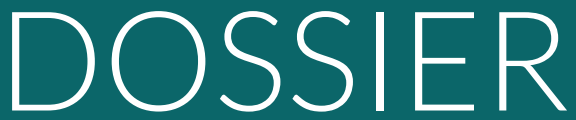

La violenza politica nell'Italia degli anni Settanta a cura di Patrizia Dogliani, Marie-Anne Matard-Bonucci 


\section{STORICAMENTE.ORG}

\section{Laboratorio di Storia}

Romain Legendre

Cosa Loro. Le discours des repentis sur la violence

Numero 10 - 2014

ISSN: $1825-411 \mathrm{X}$

Art. 22

pp. 1-28

DOI: $10.12977 /$ stor570

Editore: BraDypUS

Data di pubblicazione: 09/10/2014

Sezione: Dossier. La violenza politica nell'Italia degli anni Settanta, a cura di Patrizia Dogliani, Marie-Anne MatardBonucci 


\title{
Cosa Loro. Le discours des repentis sur la violence
}

\author{
ROMAIN LEGENDRE \\ Université Paris 8, Ecole Doctorale Pratiques et théo- \\ ries du sens, 2 rue de la Liberté, 93526 Saint-Denis
}

Cedex, France.

I "pentiti" (o "collaboratori di giustizia") contribuirono concretamente alla lotta contro la mafia siciliana. In ogni caso, il loro discorso obbedisce a codici interni all'organizzazione, basati in particolare su un'etica della violenza, oltre che legittimati dalla convergenza di interessi tra Cosa Nostra e una parte di membri delle istituzioni.

"Repenters", better called "collaborators of justice" contributed effectively to the fight against the sicilian mafia. However, their speech obeys internal codes to the organisation, based in particular on an ethics of the violence, besides legitimized by the convergence of interests between Cosa nostra and a part of the istitutions' members.

\section{Introduction}

Le repentir (pentitismo), la collaboration avec la justice ${ }^{1}$, dans une acception juridique plus pertinente, est une mesure mise en place à la fin des années 1970 pour les anciens membres des groupes terroristes. Elle

Cet article s'inscrit dans le cadre d'une recherche en cours sur «les figures du collaborateur de justice dans la lutte et l'imaginaire antimafia: le cas Tommaso Buscetta» (thèse de doctorat en cotutelle franco-italienne). 
a été étendue aux mafieux par la législation de 1991. Il s'agit d'une mesure d'exception adoptée principalement dans le cadre de la lutte contre Cosa nostra, la mafia sicilienne, par la suite élargie à d'autres champs d'application judiciaire dans le but de la normaliser. La thématique se prêterait volontiers à un travail incluant toutes les mafias italiennes, mais par souci de clarté on s'attachera ici aux collaborateurs de justice issus de l'organisation sicilienne. La notion même de discours devra être entendu au sens foucaldien. Dans cette acception sont favorisées les structures de sens implicite, indirectes, autoréférentielles, procédurales, qui ont donc tendance à se présenter comme «objectives», "partagées», «nécessaires» et «indiscutables» [Foucault 1969; 1975].

Il convient tout d'abord de définir avec précision le vocabulaire qui sera par la suite employé. Le terme de «repentir» qui sous-entend la volonté de racheter une faute morale n'apparaît nullement dans le code pénal italien. Servant dans un premier temps à qualifier ceux qui avaient renoncé à la lutte armée, reconnaissant au passage un activisme contreproductif, il fut ensuite repris pour les mafieux ayant choisi de parler à la justice. C'est dans ces circonstances que la figure, et surtout sa dimension éthico-morale, fit le plus débat. Il apparaissait pour beaucoup inconcevable qu'un mafieux poursuivant des objectifs purement criminels pût faire preuve d'une quelconque repentance. De fait, un collaborateur de justice comme Tommaso Buscetta affirma dès sa prise de parole ne pas être un repenti, attribuant au terme une connotation péjorative [Dino 2006b]. Certains, comme Pietro Aglieri et Gaspare Spatuzza ont cependant fait montre d'une volonté de reconversion spirituelle. Nous reviendrons plus avant sur les termes de la loi encadrant la collaboration avec la justice.

À l'issue de la seconde guerre mafieuse (1981-1983), qui s'apparente davantage à un massacre à sens unique, les Corleonais ont pris le contrôle de l'organisation. Il faut entendre par là tous les clans ralliés à Totò Riina et Bernardo Provenzano, et pas seulement la Famille (terme qui désigne un ancrage territorial et non une filiation) de Corleone. Salvatore Riina 
prit l'ascendant sur l'organisation et se présenta en quelque sorte comme un leader charismatique [Armao 2000], figure par ailleurs récurrente dans l'Histoire de l'Italie. Il convient toutefois de nuancer son importance tant «l'opinion publique, les politiques et les journalistes et, parfois, même les enquêteurs adorent imaginer un chef tout puissant (capo dei capi) tirant les ficelles dans l'ombre et décidant de tout» [Lupo 2010, 5]. Le bilan de ce conflit interne est resté longtemps incertain [Chinnici, Santino, 1991], ce déchaînement de violence est en tous les cas à l'origine des premières défections, à partir de 1984. Il faut cependant rappeler que le dialogue entre mafieux et forces de police a toujours existé. Dès la fin du XIXe siècle, des phénomènes de collaboration sont attestés, qui permirent la tenue de procès [Lupo 1993]. De la même manière, Paolo Pezzino notait que «le personnage de la mafia «qui parle» est beaucoup plus ancien qu'on ne le dit, puisqu'au cours des années 70 du siècle dernier [XIXe siècle] de nombreux procès de mafia se basaient sur les confessions de membres d'associations criminelles» [Pezzino 1991, 424]. Les travaux d'Umberto Santino en font également état. Au nombreux témoignages lors de procès s'ajoutait la figure du confident, source régulièrement invoquée par la police et qui fut popularisée par le roman de Leonardo Sciascia, Le jour de la chouette (1961). Le rapport «161» (13 juillet 1982), résultat des efforts combinés de la squadra mobile et du Pool antimafia de Palerme, se basa en partie sur les aveux de criminels en rapports étroits avec l'organisation ou de simples soldats de Cosa nostra. Il permit de reconstituer en partie les dynamiques internes à l'organisation mafieuse et dès lors le caractère confidentiel des sources disparut progressivement, ce qui n'empêcha pas les nombreuses polémiques sur leur instrumentalisation réelle ou prétendue.

Les collaborations de deux mafieux, Tommaso Buscetta et Salvatore Contorno, vinrent ensuite renforcer l'acte d'accusation du premier Maxi-procès intenté à Cosa nostra reconnue dès lors comme une organisation criminelle structurée de manière pyramidale (février 1986-décembre 1987). Cette logique accusatoire fut confirmée par la cour de 
Cassation en janvier 1992. Salvatore Lupo précise à ce sujet que «si cela a été la mafia à un certain moment de son histoire, cela ne signifie pas qu'elle fut semblable avant et qu'elle l'est encore aujourd'hui [...] dans la longue histoire de la mafia palermitaine, des phases de centralisation et de décentralisation se sont cycliquement alternées [...]» [Lupo 2010, 12]. Suite à la prise de contrôle des Corleonais, la mafia avait opté pour une stratégie d'attaque frontale envers l'Etat italien, le contraignant de fait à réagir. Pour autant cette réaction n'exclut pas l'existence de convergence d'intérêts entre mafia et classe politique dans l'assassinat de représentants de l'État. Il ne faut pas non plus éluder les assassinats de certains de ces représentants ayant eu lieu auparavant et avec l'assentiment de toutes les Familles. Notons par ailleurs que «la stratégie des Corleonais visait probablement à faire coïncider la réalité de l'organisation avec sa légende: une super-organisation avec un chef tout puissant» [121]. Dans la réalité, la mafia s'apparentait davantage à une république confédérale (selon l'expression d'Umberto Santino), un «ensemble d'organisation territoriales» indépendantes les unes des autres mais ayant en commun de pouvoir accéder à certaines affaires grâce à des capitaux et un réseau de relations ad hoc [Lupo 2010]. Raimondo Catanzaro note quant à lui que «l'exigence d'étoffer en permanence l'étendue des réseaux de relations sociales renforce l'impossibilité de créer des structures organisationnelles stables, accentuant ainsi le caractère fluide de l'organisation [...] la mafia n'est pas une association unique mais un système d'alliances profondément ancré socialement et disposant de liens étroits avec le système de pouvoir politique» [Catanzaro 1988, 57]. La prise de contrôle des Corleonais provoqua donc une rupture qui se manifesta par un déchaînement de violence.

Une nouvelle vague de collaboration vit le jour en 1992 suite à une nouvelle escalade terroriste et plus particulièrement après les attentats contre les juges Falcone (23 mai 1992) et Borsellino (19 juillet 1992) et à la réponse étatique qui s'ensuivit. Avant même ces attentats et suite aux maxi-procès successifs deux importants trafiquants de drogue de l'orga- 
nisation choisirent également de se ranger du côté de l'État: Francesco Marino Mannoia (1989) et Gaspare Mutolo (1992), ainsi que le mafieux de Catane Antonino Calderone (1988).

Selon Salvatore Lupo, du fait de l'escalade de la violence durant les «années de Plomb», «il fut facile [...] de comprendre que la violence créé un consensus, qu'elle permet d'exercer le pouvoir et de traiter avec, en somme que la violence paye» et c'est durant le passage entre les années soixante-dix et quatre-vingts "que se réalisa un effet de démonstration beaucoup plus vaste, comme une contagion entre deux sphères, la violence politique [des "organisations armées de la gauche révolutionnaire» et de la droite néo-fasciste] et la violence criminelle; comme un échange de modèles, de valeurs [...] entre les deux: un phénomène complexe et tortueux [...]. Tel fut le contexte culturel et politique dans lequel les mafias se renforcèrent». C'est le déchaînement de violence, notamment de Cosa nostra, qui fut «le signal le plus dramatique» de ce renforcement. Reste que cette «hybridation de la violence» avait des fins également communicatives [Dino 2006b]. Les échanges ne se limitèrent pas non plus à la dimension symbolique puisque les mafias se présentèrent comme des intermédiaires entre l'État italien et ces organisations armées révolutionnaires. Cosa nostra offrit en tous les cas "le visage ambivalent d'une organisation conservatrice venant à se comporter comme une organisation révolutionnaire, par la méthode et non par l'idéologie ou la finalité» [121]. Ce tournant a pu contribuer à la diffusion d'une certaine image de la mafia, celle d'un «contre-état, capable de proposer la négociation ou la terreur». Notons enfin que les instruments répressifs élaborés durant les «années de Plomb» furent réutilisés contre les mafias (prisons de haute sécurité, incarcérations à l'isolement, maxi-procès) tandis que certains membres des institutions participèrent à la lutte contre la mafia après avoir combattu le terrorisme (le juge Gian Carlo Caselli et le général Carlo Alberto Dalla Chiesa par exemple).

À travers cette présentation on s'attachera à expliquer de quelle manière s'articule le discours des mafieux sur la violence. On s'intéressera ainsi 
aux stratégies discursives mises en place par ces criminels à travers leur collaboration avec l'État. Leur discours à usage interne devenant dès lors un discours public entrant dans une caisse de résonance médiatique, remis en forme selon des critères subjectifs qui tiennent beaucoup à un imaginaire social sur la mafia. On entend par imaginaire social un «système cohérent, dynamique, de représentations du monde social, une sorte de répertoire des figures et des identités collectives dont se dote chaque société à des moments donnés de son histoire» [Khalifa 2013, 20]. On se demandera donc comment ces mafieux, dotés de valeurs fondées sur l'exaltation de la violence s'adressent à de nouveaux interlocuteurs. Ce phénomène de collaboration a notamment donné naissance à un genre éditorial, le livre-confession, dont la large diffusion pose la question de l'inévitable contamination des discours. On peut légitimement se demander si, dans un processus de circularité des influences, la parole des collaborateurs ayant fondé ce genre éditorial ne s'est pas finalement structurée pour devenir conforme à certaines attentes. Ce phénomène de circularité apparaît éclairant mais son analyse reste néanmoins complexe.

Après avoir présenté le contexte de production de ces discours, on évoquera les distinctions développées au travers des différents témoignages, en se demandant dans quelle mesure elles participent d'un processus de légitimation a posteriori. A travers différentes citations ont tentera de dresser une typologie des argumentaires et de dégager d'éventuelles différences entre les vagues successives de collaboration. On se demandera enfin si ces discours prennent la forme d'une contre-violence encadrée par l'État, soit une violence institutionnalisée qui se pose comme une réponse à une violence mafieuse, exercée puis subie par les collaborateurs de justice, bien souvent victimes des vengeances transversales. De leur basculement naît en effet une imbrication nouvelle des phénomènes de violence, qui a un impact sur la société civile. 


\section{Le contexte de production d'un discours sur la violence}

«Les exagérations des médias et la mauvaise connaissance de la mafia n'aident pas à comprendre le phénomène du repentir», Lupo 1993, 296. Notons tout d'abord que la loi sur les collaborateurs de justice fait totalement abstraction de la notion de repentir. Il s'agit d'un terme journalistique, repris par la suite par nombre d'acteurs judiciaires. L'État italien n'a jamais posé la repentance comme condition préalable à la collaboration. L'usage même du terme est le produit d'une confusion sémantique². La loi n. 82 du 15 mars 1991 est le résultat d'une longue maturation, elle vit le jour malgré de nombreuses polémiques, dans une atmosphère d'urgence comme c'est souvent le cas pour la lutte contre la mafia. Dans les faits, elle reprit en grande partie les normes établies par la loi Cossiga de janvier 1980, entrée en vigueur pour permettre l'abandon de la lutte armée de la part des membres d'organisations révolutionnaires (les Brigades Rouges en tête). Entre-temps, la loi du 15 novembre 1988 avait attribué au Haut Commissaire pour la coordination de la lutte contre la mafia «des pouvoirs génériques permettant de prendre des mesures de protection afin de préserver l'intégrité de ceux exposés par leur témoignage contre les organisations criminelles» [Gruppo Abele 2005, 112] (collaborateurs et témoins de justice donc, les premiers étant d'anciens mafieux tandis que les seconds sont des citoyens lambda ayant assisté à un crime perpétré par un ou plusieurs membres d'une organisation criminelle). Un programme de protection inspiré du modèle américain fut finalement mis en place avec la loi de 1991. Un service central de gestion vit également le jour, sous la tutelle du ministère de l'Intérieur. Outre la protection du collaborateur et de ses proches, la loi prévoyait en substance des réductions de peine (une condamnation à perpétuité étant par exemple commuée en peine de 12 à 20 ans de prison) en contrepartie de

Pour une analyse plus aboutie sur les différenciations entre pentitismo et pentimento, voir Dino 2006. Pour une approche analytique similaire au sujet des «années de Plomb», voir Sommier 2000. 
révélations sur les organisations criminelles. La loi fut modifiée en date du 13 février 2001 (n. 45). Entre autres changements, le collaborateur dispose désormais de 180 jours pour transmettre toutes les informations en sa possession.

A travers la collaboration, l'État entend briser un cercle de violence en substituant à la vendetta traditionnelle la justice, ce qui est fondamental car, selon le collaborateur Antonino Calderone, «un homme d'honneur ne demande justice à personne, surtout pas à l'État, il doit être capable de se faire justice lui-même» [Arlacchi 1992, 189]. Dans un tribunal où la phase de débat doit permettre la constitution de la preuve, les collaborateurs sont en mesure de révéler tout ce qu'ils savent. Ils se posent également comme des victimes d'une violence qu'ils ont par ailleurs euxmêmes pratiquée et se replacent finalement du côté de l'État. Il s'agit en quelque sorte d'un second rite de passage après celui de l'affiliation à Cosa Nostra. Pour l'État italien l'usage des collaborateurs de justice s'inscrit aussi et surtout «dans une logique pragmatique de résultats répressifs notables» [Dino 2006b, XVI].

La première vague de collaboration était composée de mafieux "perdants» à l'issue du second conflit interne à l'organisation. Craignant pour leur vie ils choisirent de se ranger du côté des institutions. La stratégie d'attaque frontale envers l'État participa d'un déchaînement de violence qui parut incontrôlé. Surtout, elle rendit l'organisation plus visible et la réponse étatique se fit évidemment plus forte. Cosa nostra choisit de fait de donner la preuve de son existence en recourant aux attentats à la bombe, on peut donc parler d'une nouvelle stratégie de communication. L'organisation, qui peut être décrite comme «relativement» secrète,

Pour une analyse détaillée des textes de loi relatifs à la collaboration d'anciens mafieux, voir Riolo 2006. Voir également l'ouvrage collectif réalisé par le Gruppo Abele 2005.

4 Mafieux de la Famille de Catane, il se décida à collaborer en 1987. Il fut arrêté dans le sud de la France, où il s'était réfugié suite à l'assassinat en 1979 de son frère, Giuseppe «Pippo»Calderone, lui aussi mafieux. Il fit notamment des révélations au sujet de la mort d'Enrico Mattéi, président de l'Ente Nazionale Idrocarburi (ENI). 
c'est-à-dire dont le caractère secret est relatif [Simmel 1996], décidait de se donner à voir de manière éclatante. A ce sujet, Salvatore Lupo a rappelé que «la société secrète joue toujours sur deux tableaux [...] si elle s'organise en secret, elle sait par ailleurs qu'elle pourra jouir d'une certaine impunité en obtenant un consensus dans l'opinion publique. Pour cela elle reste attentive à ce que son langage interne se maintienne en fluide communication avec l'extérieur» [Lupo 2008, 226]. L'impunité apparaissant notamment comme une forme de légitimation.

Suite aux conclusions du premier Maxi-procès et avant les attentats contre Falcone et Borsellino, trois mafieux de premier plan décidèrent nous l'avons dit de collaborer, permettant la tenue de nouveaux procès malgré le démantèlement du pool antimafia de Palerme. Si Calderone appartenait à un clan vaincu, Mutolo et Mannoia, très impliqués dans le trafic de drogue, faisaient eux partie des clans vainqueurs. Gagnés par le doute et craignant pour leurs vies ils décidèrent néanmoins de collaborer. C'est principalement l'engagement des membres du pool antimafia de Palerme qui permit ces défections.

La troisième vague de collaboration, après les attentats de 1992, regroupait quant à elle de nombreux affiliés gagnés par le doute suite au basculement définitif vers un terrorisme mafieux. Convaincus que Cosa nostra ne pouvait plus assurer leur protection mais au contraire décider de leur fin à la moindre désobéissance, ils optèrent finalement pour l'État, prenant acte de sa volonté apparente d'en finir avec la mafia sicilienne. Tels furent tout du moins les arguments invoqués. Il convient évidemment d'en déceler la forte dimension instrumentale. Ils purent également prendre en considération les conditions de la collaboration suite au pas franchi par leur prédécesseurs, conditions peu restrictives du point de vue du contenu des discours. Ils connaissaient par ailleurs ces discours déjà tenus sur la mafia et sur la violence en général. Cette description de la violence découle avant tout d'une perception de la mafia comme «hyper-puissance» de la part des collaborateurs de justice, perception qu'ils transmettent «essayant de nous faire raisonner avec leurs instru- 
ments interprétatifs de boss et soldats» de l'organisation [Lupo 2010, 153].

\section{Des stratégies discursives basées sur une éthique de la violence?}

D’une manière générale, plus le mafieux est en mesure "d'exercer efficacement la violence, plus il se hisse en haut de l'échelle de l'honneur [...]. Pour assumer la fonction de médiation, le mafieux [doit] bénéficier d'une réputation sociale, il [doit] être considéré comme un homme de respect; et cette considération sociale vient d'une habilité démontrée dans l'usage de la violence. [En définitive] l'exercice de la violence n'est pas une fin en soi, mais l'instrument pour l'accomplissement d'une accumulation, de telle manière que le comportement mafieux trouve dans l'enrichissement illicite une raison d'institutionnalisation» [Catanzaro 1988, 44]. Ce recours à la violence à des fins de contrôle social trouve son ancrage dans l'Italie pré-unitaire, il permit en Sicile l'affirmation du pouvoir et la conquête de richesses, en somme la mobilité sociale [Catanzaro 1988; Matard-Bonucci 1994]. Il convient donc d'appréhender cette violence dans le temps long de l'Histoire puisque, loin d'être un résidu du passé, la mafia est «un produit moderne du rapport entre le processus de formation de l'État national italien et les caractéristiques de la Sicile comme périphérie» [Catanzaro 1988, 83], elle est donc "fille de la modernité étatique» [Matard-Bonucci 1994].

Lors de la première vague de collaboration, Buscetta et Contorno affirmèrent que les valeurs de la mafia avaient été trahies au moment de l'avènement des Corleonais. Leurs discours faisant grand usage du terme «dignité» étaient emprunts de scories typiquement mafieuses, établissant une éthique de la violence. Comme l'ont démontré de nombreux chercheurs, ils s'attachèrent à décrire une ancienne mafia, selon eux bienfaitrice, pratiquant une violence légitime, en opposition à une nouvelle 
mafia enrichie par le trafic de drogue. Cette distinction était du reste couramment relayée par la presse, se basant elle-même sur des enquêtes parlementaires et judiciaires, et ce bien avant leur collaboration. Il convient donc ici de rappeler que l'organisation s'adonna à un tel trafic dès les années 1950 et que Buscetta fut très actif dans ce secteur. Il est par ailleurs exact que l'enrichissement grâce au trafic de drogue eut pour conséquence d'accentuer la conflictualité interne à l'organisation tandis qu'il provoqua un conflit violent avec l'État.

Antonino Calderone distinguait tout d'abord les homicides communs des homicides mafieux, parlant d'une violence de normale administration: «s'il faut éliminer un homme sur le territoire d'une famille pour punir une erreur normale, d'ordinaire administration, comme une confidence à la police [sbirritudine], le représentant décide, le chef de dizaine fait suivre, et cet homme n'est plus» [Arlacchi 1992, 27]. L'important étant selon lui de savoir qui était tué, pour quelle raison et par quelle Famille mafieuse, afin de maintenir les équilibres internes à Cosa nostra: "Si on ne sait pas qui a tué quelqu'un ou si on le sait d'une manière erronée alors plus personne n'est sûr de rien, pas même de sa propre vie. Et c'est ce jeu qu'ont pratiqué ces diaboliques, ces malhonnêtes Corleonais. Ils ont menti sur les homicides qu'ils faisaient» [Arlacchi 1992, 28]. Il fit toutes ces déclarations dans le cadre d'un ouvrage-entretien.

Tommaso Buscetta ${ }^{5}$ reste le collaborateur de justice le plus important puisqu'il révéla en 1984 la structure de la mafia sicilienne, parlant d'une Commission réunissant les principales Familles et prenant des décisions

Mafieux de la Famille Porta Nuova (Palerme), impliqué dans le trafic de drogue entre l'Amérique du Sud et les États-Unis. Arrêté au Brésil le 22 octobre 1983, il fut extradé vers l'Italie l'année suivante et se décida à collaborer avec la justice. Il fut d'un point de vue judiciaire le premier du genre et ses révélations contribuèrent grandement à la tenue du premier Maxi-procès de Palerme en 1986. Il dévoila notamment la structure organisationnelle de Cosa nostra. On parla à ce sujet de "théorème Buscetta». Il témoigna également lors du procès de la "Pizza connection" à New York et bénéficia de la protection des services fédéraux américains. Il est mort aux Etats-Unis le 2 avril 2000. 
impliquant tout l'organisation. La presse parla de Cupola et ce terme fut par la suite repris par certains collaborateurs. Buscetta fut aussi le plus médiatique, devenant le collaborateur «par antonomase» [Dino 2006b], affublé par ailleurs de divers surnoms. De nombreux ouvrages lui furent consacrés, dont trois prenant la forme d'entretiens écrits en grande partie à la première personne (en 1986, 1994 et 1999). Le premier, écrit par Enzo Biagi, est à l'origine du genre éditorial déjà évoqué.

Buscetta, personnage charismatique selon ses propres dires mais aussi ceux de ses divers interlocuteurs, apparaît par ailleurs intéressant car ses affirmations font entrevoir une véritable apologétique mafieuse. Il affirmait ainsi que de nombreuses idées de Cosa nostra étaient défendables mais que les Corleonais les avaient perverties et par là même détruites. Les valeurs originelles auraient été trahies, provoquant selon lui «la violence gratuite, la traîtrise, le double jeu, les trahisons et les assassinats sans réel motif». Il ajoutait plus loin au sujet de la mafia: «Elle est née pour défendre les plus faibles face aux abus des puissants et pour affirmer les valeurs de l'amitié, de la famille, du respect de la parole donnée, de la solidarité et de l'omertà, en un mot le sens de l'honneur [... . La violence gratuite et sournoise, la tromperie, le double jeu sont venus après [...] dans les années 70 et 80 [...] avec la trahison des anciennes valeurs [...]» [Arlacchi 1994, 11-12]. Toutes ces affirmations permirent à Enzo Biagi d'écrire avec son emphase habituelle: «la justice de Buscetta n'est pas la nôtre» ${ }^{6}$. Dans les faits, le collaborateur de justice produisit des discours d'autorité influençant fortement les magistrats et les journalistes.

Dans les faits, la violence mafieuse est à la fois socialisante et «excluante», puisque la capacité à se conformer à des standards de violence ou bien plus encore à les surpasser, permet de «faire carrière» dans l'organisation, tandis que ceux qui se montrent incapables d'une telle audace sont plus généralement en retrait ou bien demeurent tout simplement victimes de leurs concurrents. La violence est imposée comme une «norme de

Biagi E., Tre notti con il boss, «Panorama», 19 ottobre 1986. 
régulation de la compétition de marché» [Catanzaro 1988, 260]. Elle est à la base du pouvoir mafieux dont les fonctions sont de nature économique et politique, elle est d'abord présentée comme une menace, avant d'être exercée si nécessaire. L'historien Eric Hobsbawm l'a donc théorisée comme «extorsion contrôlée».

Salvatore Cucuzza ${ }^{7}$, qui collabora à partir de 1992, reprit une partie des arguments de Buscetta en affirmant que, "contrairement à ce qui se dit la mafia d'alors était contre le crime». La généricité de tels propos est éclairante car en ne situant pas précisément cet "alors", le collaborateur renvoie de fait à un temps non-historique et révèle par là même le caractère instrumental de ce discours, l'invention d'une tradition. Chaque tradition est «inventée», c'est-à-dire qu'elle dérive d'une réélaboration instrumentale du passé opérée dans le présent et à des fins présentes [Hobsbawm, Ranger 1983]. Ici en l'occurrence cette «invention» sert à justifier l'appartenance à la mafia et l'existence même de l'organisation. Salvatore Contorno , qui collabora concomitamment avec Buscetta, partageant la même cellule à Rome lors des premiers mois, résuma la situation de la manière suivante lors du premier Maxi-procès: «Cosa nostra est devenue Cosa nostra personnelle». Manière d'expliquer que l'organisation ne défendait plus les intérêts communs selon lui. Tout en prenant avec beaucoup de distance ces considérations, il faut rappeler que les Corleonais s'en prirent aux familles de leurs ennemis en général

7 Mafieux de la Famille Borgo di Palermo, il débuta sa collaboration en 1996. Il fit des révélations sur le meurtre de Pio La Torre et sur la «strage della circovallazione» durant laquelle moururent le boss catanais Alfio Ferlito et trois carabiniers. Il a également permis la confiscation de biens immobiliers pour une valeur de plusieurs millions d'euros.

8 Mafieux de la Famille de Santa Maria del Gesù. Avant de devenir collaborateur de justice, il agit dans un premier temps comme confident auprès d'un policier palermitain. Réfugié à Rome pour échapper à la mort il fut arrêté et entreprit alors de collaborer. Il permit aux enquêteurs de reconstituer certaines dynamiques de la seconde guerre de mafia et décrit également la structuration de Cosa nostra lors du premier Maxiprocès. Envoyé aux Etats-Unis pour bénéficier de la protection des services fédéraux, il revint en Sicile dans des circonstances troubles et fut de nouveau arrêté. Il effectua par la suite d'autres séjours en prison pour des délits mineurs. 
et à celles des collaborateurs de justice en particulier. Buscetta perdit ainsi douze membres de sa famille, Contorno plus d'une vingtaine. Ces vengeances transversales participèrent d'un déchaînement de violence révélant davantage que par le passé la plasticité des valeurs de l'organisation. Ce déchaînement rendit sceptiques de nombreux observateurs quant à l'existence réelle d'une structure dotée d'un organe décisionnaire. La violation permanente des règles apparaissant clairement dans les révélations des collaborateurs, il fallait en conclure, selon certains contradicteurs, que celles-ci n'existaient pas. Pourtant «le manque de conformité au code ne signifie pas que le code n'existe pas, tout comme la violation de la loi ne nous amène pas à conclure que la loi n'existe pas [...] leurs règles sont orales [...] vaguement énoncées» [Lupo 2008, 239-240] et donc d'autant plus faciles à piétiner. Toujours pour parler de ce déchaînement, Umberto Santino qualifia les actes des Corleonais au cours de la seconde guerre de mafia et par la suite, de «délire de toutepuissance criminelle» [Santino 2006, 70].

Giovanbattista Ferrante, issu de la troisième vague de collaboration, expliqua lui que la violence était appréhendée de manière pragmatique et n'effectua aucune distinction entre ancienne et nouvelle mafia. On lui aurait déclaré «ne vous inquiétez pas parce que ce n'est pas beau à voir mais on s'y habitue» [Gruppo Abele 2005, 299]. Il ajoutait «Le discours a toujours été très doux, toujours graduel». manière de parvenir à une acceptation, à un détachement de la part des affiliés. Salvatore Cancemi ${ }^{10}$ tint un discours similaire en évoquant le crime commis en préambule à son affiliation, expliquant qu'il avait été contacté pour cet

Mafieux de la Famille San Lorenzo, il collabora à partir de 1996. Il a reconnu son implication dans les attentats de Capaci et Via d'Amelio, tout en faisant des déclarations très importantes concernant les assassinats de Salvo Lima, Rocco Chinicci, Antonio Cassarà et Roberto Antiochia.

10 Chef régent de la Famille Porta Nuova, membre de la Commission de Cosa nostra, il débuta sa collaboration en 1993 et permit l'arrestation de nombreux mafieux de premier ordre. Il fut également précieux dans le cadre des procès sur les attentats de Capaci et Via d'Amelio. 
homicide comme s'il s'agissait d'un acte commun. Vittorio Mangano, membre de la Famille Porta Nuova, lui aurait déclaré «moi et quelques autres souhaitons que tu y ailles [...] la chose m'a été présentée ainsi, de manière très légère» [260].

Pour en revenir à Salvatore Cucuzza, il se décida à collaborer, affirmant ne plus partager «les aspects les plus criminels gratuits» [280]. C'est uniquement à partir du recours systématique au terrorisme qu'il opère cette distinction. Son discours semble avant tout démontrer que cette escalade dans la violence, loin d'assurer l'impunité à l'organisation finit par la rendre vulnérable. Il ajoutait «quelqu'un qui tue un bébé ne peut pas parler de sens de l'honneur». Faisant en cela référence à Giovanni Brusca. De fait, la troisième vague de collaboration permet de démontrer la fuite en avant de Cosa nostra et le recours à une violence accrue. Brusca $^{11}$, qui réalisa un ouvrage-entretien avec le journaliste Saverio Lodato en 1998, intitulé, "J'ai tué Giovanni Falcone» fit séquestrer pendant 2 ans puis étrangler et dissoudre dans l'acide le fils du collaborateur de justice Santino Di Matteo ${ }^{12}$, âgé de 8 ans, afin de le contraindre au silence au sujet de l'attentat de Capaci. Il répondit par voie de presse aux déclarations de Cucuzza en contestant la nature de son crime, déclarant au sujet

11 Mafieux de la Famille de San Giuseppe Jato, fils du boss Bernardo Brusca. Surnommé «l'étrangleur de chrétiens». Au cours de l'ouvrage-entretien avec le journaliste Saverio Lodato, il avoua quelques 150 meurtres, il fut notamment celui qui déclencha l'explosion lors de l'attentat de Capaci, dans lequel moururent Giovanni Falcone, sa femme Francesca Morvillo et trois membres de leur escorte. Arrêté en 1996, il entreprit aussitôt une collaboration qui se révéla instrumentale, ce qui lui valut d'être inculpé pour calomnie. Il fut néanmoins réintégré au programme de collaboration et obtint des contreparties comme la liberté conditionnelle en échange de certaines informations. Il a récemment été interrogé dans le cadre du procès actuellement en cours à Palerme, concernant des complicités entre Cosa nostra et des membres des institutions. Sa collaboration est certainement la plus polémique étant donné l'ampleur des crimes commis et les modalités de ses prises de parole successives.

12 Membre de la Famille d'Altofonte, il fut arrêté en 1993 et décida de collaborer avec la justice la même année. Il fut l'un des artificiers de l'attentat de Capaci et témoigna à ce sujet. 
de l'enfant: «je n'ai pas tué un bébé. Allez voir la date de naissance»'3. D'une manière générale, ce qui caractérise la mafia c'est sa capacité à se transformer, à opérer des mutations dans la continuité. La distinction entre ancienne et nouvelle mafia est donc factice. Il est avéré en revanche que la tactique des Corleonais créa un sentiment d'insécurité dépassant finalement le bénéfice retiré du statut de mafieux. Et c'est avant tout comme cela qu'il faut l'appréhender. Paradoxalement, certains affiliés furent gagnés par la crainte qu'ils avaient auparavant inspiré, redoutant l'exécution par un clan adverse ou l'arrestation. Gaspare Mutolo', qui collabora à partir de 1992, avoua ainsi «j'avais peur [...] peur d'être assassiné, même en prison [...] j'avais peur pour ma famille»'s. Dans tous les cas une certaine éthique de la violence se fait jour, qui évolue sensiblement entre les différentes vagues de collaboration. Le caractère instrumental apparaît avec évidence dans la mesure où les discours évoluent en fonction des évènements. Il ne faut cependant pas exclure un conditionnement politico-culturel des affiliés. L'éducation à être mafieux permet la croyance dans les valeurs de l'organisation définie comme "forme culturelle, historiquement conditionnée, d'agissement politique». La mafia se présente ainsi comme un ordre social et culturel, système de symboles et de rituels qui diffuse des idées et des concepts sur la nature de l'ordre social et l'existence humaine, en se réappropriant des valeurs communes qui font sens» [Santoro 2007, 27] et permettent par là même l'obtention d'un consensus social [Santino 2006]. Comme l'a rappelé la sociologue Alessandra Dino, «le choix d'un symbole de la part d'un sujet est lié à une volonté précise d'insérer ses agissements dans un univers de significations bien défini, duquel sont

13 «Corriere della Sera», 21 janvier 1997: Di Piazza 2010, 50.

14 Mafieux de la Famille Partanna-Mondello, il débuta sa collaboration en 1992, il a reconnu plusieurs meurtres et révélé de nombreux aspects du trafic d'héroïne. Il a récemment témoigné dans le cadre du procès qui se tient actuellement à Palerme.

15 Marcelle Padovani, Mafia italienne. Le crépuscule des repentis, «Le Nouvel Observateur», 14 octobre 2011. 
utilisés et mis en commun des répertoires d'action bien précis» [Dino 2010, 240]. C'est notamment dans cette optique qu'il faut analyser les fondements religieux d'une éthique sur la violence, qui tend à élever le mafieux au niveau de Dieu. En définitive, les mafieux estiment exercer une forme de violence divine lorsqu'ils commettent des homicides $[35]^{16}$. Ils se dotent par ailleurs d'une nouvelle identité qui s'accompagne de la création de liens de fraternité rituelle [Paoli 2000; Dino 2010]. Le collaborateur Antonino Calderone affirmait ainsi: "nous sommes des mafieux, les autres ne sont que des hommes» [Arlacchi 1992, 12]. Cette radicalité constituerait à la fois la force et la faiblesse de Cosa nostra. Lorsqu'ils cessent de croire en ces valeurs, pour diverses raisons, les mafieux peuvent en effet être profondément affectés et décider dans le même temps de rompre de manière définitive avec l'organisation pour se tourner vers l'État [Dino 2006b]. Ainsi Francesco Marino Mannoia ${ }^{17}$ déclara «j'ai décidé de collaborer avec la justice après une longue réflexion car je suis fatigué d'une appartenance à Cosa nostra qui m'a causé un grave trouble intérieur et une profonde crise de conscience» ${ }^{18}$.

Cependant, les mafieux ayant collaboré suite aux attentats de 1992 évoquèrent pour la plupart une erreur stratégique à travers le recours au terrorisme mafieux. Salvatore Cucuzza affirma ainsi: «La fin de Cosa Nostra a débuté avec la mort des deux magistrats, Falcone et Borsel-

Nous renvoyons à l'ouvrage de la sociologue pour une analyse détaillée du discours
religieux employé par les mafieux.
17 Membre de la Famille Santa Maria del Gesù, il fut épargné lors de la seconde guer-
re de mafia car il se trouvait en prison pour trafic international d'hérö̈ne. Il officiait en
effet comme chimiste. Une fois évadé de prison en 1983, il rallia le clan des Corleonais.
De nouveau arrêté en 1985, il décida de collaborer en 1989 suite à l'assassinat de son
frère Agostino. Sa prise de parole lui valut de perdre sa mère, sa sœur et sa tante. Il fut
le premier collaborateur issu des clans vainqueurs. Parmi ses révélations, il faut surtout
évoquer celle relative aux rencontres entre son boss, Stefano Bontate et le sénateur Giu-
lio Andreotti. Ses affirmations ont contribué à reconnaître la culpabilité de l'homme
politique, condamné pour association de malfaiteurs mais bénéficiant finalement de la
prescription des faits. Pour une analyse de cette affaire nous renvoyons à Briquet 2007 . 18 Article non signé, La "cantata” di Mannoia, «L’Ora», 6 décembre 1989. 
lino. Là s'est terminée Cosa nostra, parce que le consensus populaire qu'elle avait avant était très fort, après cela a été terminé, complètement» [Gruppo Abele 2005, 279]. Au sujet de l'attentat contre Giovanni Falcone, Giuseppe Marchese ${ }^{19}$ déclara "quelle chose ignoble ils ont fait, ils ont fait sauter la moitié de l'autoroute, il y aurait pu y avoir d'autres morts» [314]. Ce n'est donc pas tant l'assassinat du juge qui le dérangeait, que le mode opératoire employé. Giovanni Drago ${ }^{20}$ collabora lui aussi après avoir remis en doute cette stratégie. Au sujet des crimes passés il évoqua une logique interne à Cosa nostra: «j'ai toujours tué parce qu'ils étaient mes adversaires. Si je ne l'avais pas fait moi, ils s'en seraient chargés. Je les voyais comme mes adversaires» [293]. Cucuzza et Mutolo tinrent également ce discours. Ce dernier déclara notamment «Je n'avais pas le choix. J'appartenais à une organisation où la principale vertu est l'obéissance et où les ordres doivent être exécutés. Je me sentais autorisé, légitimé même, à commettre des crimes. Mon État, c'était Cosa Nostra»"12. Là encore, une logique interne à l'organisation fut presque unanimement défendue et très largement diffusée. Les lois dressées par l'État italien ne semblent à aucun moment prises en considération dans leurs déclarations comme s'il était uniquement question de Cosa nostra, de «leur chose» (cosa loro) et qu'elle se présentait comme un anti-état.

$\mathrm{Au}$ sujet de la dénomination, on peut également noter que «dans le contexte de l'organisation mafieuse, le singulier tend à s'identifier avec le groupe et son identité tend à devenir collective», d'où le nom "notre chose» [Dino 2010, 71]. Il faut cependant rappeler que l'organisation

\footnotetext{
19 Beau-frère du mafieux Leoluca Bagarella, membre de la Famille mafieuse de Corso dei Mille alliée aux Corleonais. Il a notamment dénoncé les meurtriers de l'homme politique Salvo Lima et admis sa responsabilité dans le meurtre du policier Giuseppe Montana.

20 Membre de la Famille de Brancaccio, il collabora à partir de 1992. Il a contribué à la condamnation de tout son clan et a également témoigné contre Giulio Andreotti et lors du procès sur l'assassinat de Salvo Lima.
}

21 Padovani Marcelle, Mafia italienne. Le crépuscule des repentis, «Le Nouvel Observateur», 14 octobre 2011. 
mafieuse américaine fut également nommée de cette manière, tout en disposant d'un fonctionnement bien différent. Le fait de mettre un nom sur son ennemi permit avant tout aux autorités américaines de le qualifier auprès de la société civile au moment des révélations du collaborateur de justice Joe Valachi (1962), lesquelles firent par ailleurs l'objet d'un ouvrage très vendu, Valachi Papers, écrit par le journaliste Peter Maas (1971).

Pour en revenir à la notion d'anti-état, le lien semble en réalité inextricable entre l'organisation mafieuse et l'État italien. L'organisation n'est donc pas un anti-état mais plutôt «l'expression du politique dans la subalternité» [Santoro 2007]. Un manque de légitimité a notamment conduit l'État à l'attribution d'une fonction d'ordre à la mafia, parfois dans le but de réduire des oppositions politiques, aboutissant par là même à la remise en cause du monopole de la violence légitime qu'il était censé détenir [Weber 1971]. Ce n'est pas tant son absence qui est en cause dans le développement de l'organisation en Sicile, mais bien les modalités de sa formation et de son fonctionnement [Catanzaro 1988; Lupo 1993; Matard-Bonucci 1994].

Bien souvent il n'y a donc pas de repentance car le sentiment de légitimité prédomine. Il est cependant difficile de savoir si ces discours sont toujours le résultat d'une stratégie obéissant à de vastes raisons utilitaristes. Comment savoir si ces mafieux, en qualifiant leur appartenance, négligent consciemment ou inconsciemment l'interdépendance historique entre l'organisation et l'État italien ? Reste que, d'une manière générale, on condamne un excès de violence, pas son usage comme moyen pour arriver à une fin.

Bien que cette appréciation de l'excès de violence varie sensiblement entre les différentes vagues de collaboration, on peut observer la construction d'un certain discours qui tend à s'uniformiser, à se lisser. Au fond, les collaborateurs de justice finissent par dire ce qu'on attend d'eux à un moment donné. Ils produisent une parole sur un marché linguistique, comme si les premiers collaborateurs avaient fixé un usage légitime des 
discours, une nomenclature dont les contours auraient été également délimités par la couverture médiatique. Des pratiques «nouvelles» considérées comme une perversion des idéaux de l'organisation seraient ainsi la cause de la collaboration des mafieux. En somme, après avoir reconnu les institutions, les collaborateurs ne se contentent pas de décrire une violence mafieuse, ils persistent bien souvent à la défendre publiquement. De cette manière, l'interférence consensuelle avec l'État se situe également au niveau des discours.

\section{Un discours de contre-violence encadré par l'État?}

Le discours, les mots, restent la seule arme à disposition des collaborateurs de justice. Leur savoir sur l'organisation, énoncé clairement devant un juge, détient un potentiel de destruction. Plusieurs phénomènes de violence s'imbriquent car à travers leurs révélations ils décrivent la violence mafieuse, usant d'une phraséologie propre à l'organisation, tout en participant d'une violence étatique qui les voit bénéficier de remises de peine tandis que ceux qui ont préféré garder le silence subissent des peines exemplaires, notamment le régime 41bis, l'incarcération à l'isolement. Par ailleurs, les mafieux de haut rang ayant évidemment bien plus à révéler, ils sont en théorie favorisés dans le cadre des contreparties prévues pour leur collaboration, au détriment des simples soldats de l'organisation. Ces dispositions légales ont provoqué de nombreux débats sur l'égalité de la peine, et démontrent un autre aspect d'une médiation informelle sans cesse à l'œuvre entre les organisations criminelles et l'État.

A travers la collaboration se pose la question du contrôle du discours. Tout d'abord parce que les mafieux «usent d'un langage qui abuse des potentialités symboliques du dialecte» [Di Piazza 2010, 39], langage par nature moins codifié que les langues officielles. Or la langue officielle a partie liée avec l'État. À ce titre, le dialecte apparâit comme un langage 
illégitime du point de vue juridique et judiciaire. Le code pénal, surnommé code Rocco, (en vigueur de 1930 à 1988, remplacé par la suite par le code Vassalli) stipulait de fait que les dépositions au cours d'interrogatoires et pendant les audiences devaient être effectuées en italien. Se posait dès lors la question de l'authenticité. Le cas se présenta de manière concrète lors du témoignage de Salvatore Contorno au cours du premier Maxi-procès. Il décida en effet de s'exprimer dans un dialecte sicilien très fermé, choix stratégique lui permettant de signifier qu'il réglait ses comptes avec ses anciens coreligionnaires. "J'ai été contraint de devenir un traître comme ils m'appellent. Mais ce sont eux les traîtres. Ce n'est pas moi !» Affirma t-il notamment lors d'une audience. Il faut noter qu'il abandonna cette stratégie lors des procès suivants, s'exprimant dès lors uniquement en italien bien qu'il ait affirmé dans un premier temps ne pas le maîtriser. La justice peut donc être tentée de laisser une certaine marge de manœuvre au collaborateur afin de permettre l'acquisition de la preuve mais ce faisant elle s'expose à la manipulation.

Pour en revenir aux déclarations de Contorno, il faut noter qu'il existe bel et bien des contraintes réelles ayant produit des effets de rejet ancrés dès l'affiliation à la mafia. Comme l'a expliqué Salvatore Cancemi, il est impossible de la refuser: "parce que celui-là ensuite ils doivent le tuer, ils ne peuvent pas le laisser en vie parce qu'il sait cette chose et il ne fait pas partie de Cosa nostra» [Gruppo Abele 2005, 260]. Il peut donc y avoir une dimension coercitive dans l'affiliation. L'impétrant faisant face à plusieurs personnes se voit confronté à une violence psychologique qui préfigure une violence physique en cas de refus de sa part. Le choix peut donc être contraint. Les révélations de Melchiorre Allegra ${ }^{22}$, collaborateur de justice de la période fasciste, abondent en ce sens: «A ce moment

«Repenti» de la période fasciste, il fut arrêté par les Carabiniers de Castelvetrano en juillet 1937. Il révéla alors l'existence et le fonctionnement de l'organisation appelée «mafia». Ses révélations furent publiées par le journaliste Mauro De Mauro dans le quotidien «l'Ora» en 1962. Il fut qualifié de "proto-repenti», pourtant d'autres avant lui ont parlé, tel Salvatore D’Amico. Pour toutes ces observations: Lupo 1993. 
je fus interpellé pour savoir si j’acceptais de faire partie de la mafia. Je compris que j'avais déjà été mis au courant de trop de secrets, y compris ceux concernant l'activité criminelle, et que je ne pouvais pas ne pas accepter si je voulais sortir vivant de cette réunion» [50].

Leonardo Vitale ${ }^{23}$, qui reste à ce jour considéré comme le premier repenti stricto sensu de la mafia sicilienne, affirmait lui avoir été contraint de devenir mafieux par son environnement familial. Lorsqu'un oncle lui ordonna d'accomplir un homicide, il se senti obligé d'obéir. Il déclara ensuite aux policiers "on ne naît pas mafieux, ils nous le font devenir» [Stajano 1986 ; Gruppo Abele 2005, 70]. Vitale faisait en effet partie de ce qu'il convient d'appeler une dynastie mafieuse, ce qui ne fut pas le cas de tous les affiliés, ses aveux ne sauraient donc servir pour une quelconque généralisation.

Vitale comme Allegra décidèrent en tous les cas de briser le silence alors qu'ils étaient soupçonnés par les autorités dans différents délits. Leur prise de parole constitua aussi une échappatoire. Reste qu'ils prirent cette décision à des moments où le phénomène de collaboration n'était délimité ni juridiquement ni médiatiquement. Quel que soit le contexte, la démarche peut constituer une libération et s'apparenter à une forme de contre-violence, dans le sens de réponse à la violence de l'autre. Du reste, cette stratégie, à savoir de définir sa propre violence comme réponse à une autre, se retrouve en dehors de la mafia, elle a notamment été étudiée en ce sens dans la philosophie sartrienne.

Dans le droit pénal italien, un délit est également défini comme une

\footnotetext{
23 Membre de la Famille d'Altarello di Baida, en 1973 il se présenta dans une caserne de carabiniers et fit de nombreuses révélations sur la structure de la mafia. Fragile mentalement, il fut condamné et enfermé dans un asile psychiatrique à l'issue d'un procès au cours duquel toutes les personnes qu'il avait accusé furent acquittées pour insuffisance de preuves, à l'exception de son oncle, lequel l'avait introduit dans l'organisation. Ce dernier fut d'ailleurs assassiné en représailles. Surnommé le «Valachi des faubourgs» par la presse, Leonardo Vitale subit lui aussi la vendetta de la mafia. Pris dans une embuscade alors qu'il sortait de la messe, il mourut des suites de ses blessures le 2 décembre 1984. Nombre de ses révélations furent confirmées par les collaborateurs Tommaso Buscetta et Salvatore Contorno.
} 
offense voire une destruction du bien juridique et à ce titre, la collaboration s'inscrirait également dans une logique de contre-offense [Dino 2006b]. Après avoir bénéficié du pouvoir de coercition de l'organisation pour accumuler des capitaux de manière illégale, le collaborateur, de par ses révélations, permet la confiscation des biens mafieux et serait ainsi en mesure de réparer en partie l'offense.

Il est néanmoins bien souvent question de contre-violence ou de contreoffense orientée. Tous notèrent ainsi la volonté de Buscetta d'incriminer les Corleonais (responsables de l'assassinat de nombre de ses proches) tout en épargnant dans une certaine mesure Gaetano Badalamenti, boss de Cinisi auquel il fut très lié dans le cadre du trafic de drogue international. Il nia par ailleurs son implication dans un tel trafic, malgré les nombreux rapports de police à ce sujet. Les juges lui concédèrent ce point pour des raisons pratiques. Il «révéla des choses tellement importantes, tellement innovantes [...] qu'elles incitèrent les enquêteurs à ne pas insister sur l'invraisemblance de certaines de ses interprétations, en particulier celles rattachant la dégénération de Cosa Nostra à la férocité et au sadisme de Riina» [Lupo 2010, 5].

Suite à l'augmentation du phénomène, cette contre-violence fut encadrée avec peine par les structures étatiques puisque certains collaborateurs admis au programme de protection retournèrent en Sicile pour commettre des crimes mafieux (racket, trafic de drogue) et exercer des vengeances transversales envers d'autres membres de l'organisation (ainsi Balduccio di Maggio ou encore Giuseppe Ferone).

À travers leurs révélations les collaborateurs cherchaient donc à empêcher diverses formes de violence à leur encontre tout en l'exerçant sur les autres mafieux avec le concours de l'État, ou à son insu. Ce dernier, par souci d'authenticité, laissa une certaine liberté dans le contenu des déclarations, permettant ainsi la diffusion de discours sur la violence, tandis que ses limites structurelles ne parvinrent à empêcher la récidive et les entorses au programme de collaboration. Les discours furent retranscrits, mis en forme, par la magistrature puis par la presse, créant 
deux filtres d'interprétation qui peuvent légitimement faire penser que, bien souvent, une partie du sens nous échappe.

\section{Conclusion}

Les collaborateurs de justice ont permis d'acquérir une connaissance inédite de Cosa nostra, de son organisation, de ses rituels, nous donnant à voir «une violence [devenue] d'une manière programmatique instrument de profit et de lutte politique en adoptant justement les structures d'organisation adéquates à ses buts criminels» [Pezzino 1991, 425]. Mais à travers leurs révélations ils ne se départent pas d'un langage interne et par certains discours ils contribuent à la diffusion de valeurs articulées autour d'une violence tolérable/tolérée [Dino 2006a]. Le détachement de la mafia, pensée comme ordre social, s'accompagne donc de la permanence d'un attachement aux valeurs morales de l'organisation, qui participe de processus d'acculturations, de nombreux criminels de droit commun agissant en effet par mimétisme tandis que les représentations de la mafia sont connotées de manière positive pour une partie de la société civile. En somme, bien que considérés comme des «infâmes», les collaborateurs ne trahissent pas totalement l'organisation.

Leur prise de parole, faite de déclarations brutes et parfois brutales, gage apparent d'authenticité, tend également à se conformer à un discours déjà délimité, à une attente supposée ou réelle. En définitive, le discours du collaborateur, comme celui du mafieux, semble répondre à une logique d'appartenance et tend à s'adapter à un marché linguistique. Il faut noter enfin que certains ont continué de servir des intérêts factieux à travers une collaboration stratégique, comme Giovanni Brusca ou Giuseppe Ferone, tandis que d'autres ont été instrumentalisés par la magistrature et les forces de police, comme ce fut le cas pour Vincenzo Scarantino.

Plus globalement, le changement de stratégie de l'organisation, associé 
à une désaffection généralisée de la société civile pour cette figure a entraîné la diminution du phénomène à la fin des années quatre-vingt dix. Cette nouvelle stratégie s'est caractérisée par une plus grande compréhension à l'égard des collaborateurs, les incitant parfois à se rétracter, le tout dans un contexte de normalisation commode pour Cosa Nostra comme pour l'État. La politique d'enfouissement engagée par Bernardo Provenzano (1995-2006) a pu faire croire à un retour aux valeurs prétendument originelles, prises comme existantes puisque défendues par nombre de collaborateurs. Ces derniers eurent par ailleurs beaucoup moins à révéler et ce qui restait à dire concernait surtout les liens entre l'organisation et la classe politique. La contestation du phénomène se fit plus forte précisément lorsque les révélations atteignirent ce point critique. Les membres du corps d'État s'affrontèrent, une partie de la classe politique fustigeant la propension des magistrats à s'immiscer dans ses prérogatives tandis que la société civile tendait à s'identifier aux juges dans une logique d'opposition binaire, ceci bien que la magistrature soit une partie organique de l'État. L'usage considéré, à tort ou à raison, comme instrumental des collaborateurs de justice, a pu alimenter les perceptions complotistes (dietrologie) de la réalité, une perception nourrie par la couverture médiatique et les discours politiques. Toutes ces controverses ont finalement contribué à la réélaboration de la loi sur les collaborateurs de justice. Quoi qu'il en soit, même lorsque la parole sembla lui échapper, certaines des valeurs de la mafia se diffusèrent à travers la collaboration. Il ne s'agissait cependant pas d'un contrôle objectif mais d'une diffusion propre à un système culturel, par nature dynamique et aléatoire.

La mafia sicilienne ne saurait être analysée comme un antisystème et elle a pu être considérée à tort comme un hypersystème, soit un système plus important qu'un premier considéré, ici en l'occurrence l'État italien, notamment parce qu'elle créait les conditions de l'insécurité pour se présenter ensuite comme protectrice. Le recours aux attentats a provoqué une réaction étatique s'inscrivant dans une logique de l'urgence 
et il a notamment été question d'un état de guerre. Or, «'état de guerre définit les rapports entre deux sujets juridiques de même niveau, deux entités d'états homogènes, bien qu'en opposition et présuppose justement que la mafia est ainsi et que c'est de cette manière qu'il faut la considérer» [Pezzino 1991, 419-437]. En somme, les catégories interprétatives de la classe politique et de la presse ont alimenté une ambiguïté parfois destinée à éluder certaines compromissions.

\section{Sources}

Arlacchi P. 1992, Gli uomini del disonore. La mafia siciliana nella vita del grande pentito Nino Calderone, Milano: Mondadori.

Arlacchi P. 1994, Addio Cosa Nostra. I segreti della mafia nella confessione di Tommaso Buscetta, Milano: Rizzoli.

Biagi E. 1986, Il boss è solo. Buscetta: la vera storia di un vero padrino, Milano: Mondadori.

Biagi E. 1986, Tre notti con il boss, «Panorama», 19 ottobre.

La "cantata” di Mannoia, s.a., "L'Ora», 6 décembre 1989.

Lodato S. 1999a, La mafia ha vinto, Milano: Mondadori.

Lodato S. 1999b, "Ho ucciso Giovanni Falcone" la confessione di Giovanni Brusca, Milano: Mondadori.

Padovani M. 2011, Mafia italienne. Le crépuscule des repentis, «Le Nouvel Observateur», 14 octobre.

\section{Bibliographie}

Armao F. 2000, Il sistema mafia. Dall'economia mondo al dominio locale, Torino: Bollati Boringhieri.

Briquet L. 2007, Mafia, justice et politique en Italie. L'affaire Andreotti dans la crise de la République (1992-2004), Paris: Karthala. 
Catanzaro R. 1988, Il Delitto come impresa. Storia sociale della mafia, Padova: Liviana.

Chinnici G., Santino U. 1991, La violenza programmata. Omicidi e guerre di mafia a Palermo dagli anni Sessanta ad oggi, Milano: Franco Angeli. Dino A. (ed.) 2006a, La violenza tollerata. Mafia, poteri, disobbedienza, Milano: Mimesis.

Dino A. (ed.) 2006b, Pentiti. I collaborati di giustizia, le istituzioni, l'opinione pubblica, Roma: Donzelli Editore.

Dino A. 2010, La mafia devota, Roma-Bari: Laterza.

Di Piazza S. 2010, Mafia, linguaggio, identità, Palermo: Centro di studi ed iniziative culturali Pio La Torre.

Foucault M. 1969, L'archéologie du savoir, Paris: Gallimard.

Foucault M. 1975, Surveiller et punir. Naissance de la prison, Paris: Gallimard.

Gruppo Abele (ed.) 2005, Dalla mafia allo Stato. I pentiti: analisi e storie, Torino: Ega.

Hobsbawm E., Ranger T. (dir.) 2006 [1983], L'invention de la tradition, Paris, Éditions Amsterdam.

Khalifa D. 2013, Les bas-fonds. Histoire d'un imaginaire, Paris: Seuil.

Lupo S. 1993, Storia della mafia dalle origini ai nostri giorni, Roma: Donzelli Editore.

Lupo S. 2007, Che cosè la mafia. Sciascia e Andreotti, l'antimafia e la politica, Roma: Donzelli Editore.

Lupo S. 2008, Quando la mafia trovò l'America. Storia di un intreccio intercontinentale, 1888-2008, Torino: Einaudi.

Lupo S. 2010, Potere criminale. Intervista sulla storia della mafia, Gaetano Savatteri (ed.), Roma-Bari: Laterza.

Matard-Bonucci M.-A. 1994, Histoire de la Mafia, Bruxelles: Complexe.

Paoli L. 2000, Fratelli di mafia. Cosa nostra e 'Ndrangheta, Bologna: Il Mulino.

Pezzino P. 1990, Una certa reciprocità di favori. Mafia e modernizzazione 
violenta nella Sicilia postunitaria, Milano: Franco Angeli.

Pezzino P. 1991, La modernisation violente en Italie: perspective historique du crime organisé, "Déviance et société», (4): 419-437.

Riolo S. 2006, La legislazione premiale antimafia, in Dino 2006b, 3-38.

Santino U. 2006, Dalla mafia alle mafie. Scienze sociali e crimine organizzato, Soveria Mannelli: Rubbettino Editore.

Santoro M. 2007, La voce del padrino. Mafia, cultura, politica, Verona:

Ombre corte.

Simmel G. 1996, Secret et sociétés secrètes, Paris: Éditions Circé.

Stajano C. (dir.) 1986, Mafia. L'atto d'accusa dei giudici di Palermo, Roma:

Editori Riuniti.

Weber M. 1971, Économie et société, Paris: Plon. 